\title{
NETWORK-BASED SCIENTIFIC COMPUTING
}

\author{
Elias N. Houstis, Ann Christine Catlin, Ganesh Balakrishnan, \\ Nitesh Dhanjani, GaHyun Park, John R. Rice \\ Purdue University \\ West Lafayette, IN, USA
}

\author{
Spyros Lalis, Manolis Stamatogiannakis \\ Foundation for Research and Technology \\ Heraklion, Crete, Greece
}

Catherine Houstis

University of Crete

Heraklion, Crete Greece

\begin{abstract}
Rapid advances in modern networking technologies and commodity high performance computing systems are leading the field of computing in a new paradigm referred to as network-based computing (NC). This paradigm views a large number of geographically distributed computer resources such as PCs, workstations, Symmetric Multi-processors (SMP) and Massively Parallel Processing (MPP) systems connected through a high speed network as a single meta-computer or computational grid [2]. In this paper, we focus on the Internet (WAN) and Intranet (LAN) based computational grids and their ability to support scalable and robust "deep" computing. We present various implementations of the NC paradigm using commodity and customized software in the context of the existing PELLPACK problem solving environment [8], the ITPACK library as it has been implemented in the PELLPACK system, and a muti-physics application for the design of gas turbine engines [11]. Through this study we attempt to assess the feasibility and efficiency of several NC paradigms for scientific applications utilizing existing middleware.
\end{abstract}

Keywords: network computing, grid computing, deep computing, problem solving environment, legacy code, middleware, agent based computing, clientserver computing, multi-physics application. 


\section{INTRODUCTION}

The Internet has long been used for communication. Until recently, there has been little use of the network for actual computations. This situation is changing rapidly and will have enormous impact on the future. It is clear that the old slogan "The Network is the Computer" is becoming a reality. It will not only change the way we live, work, learn, and communicate with each other, but it will change the way we do computational science and electronic prototyping. In this paper we assess the capability and effectiveness of several commodity and customized software technologies to support scalable and robust "deep" scientific computing on Internet and Intranet based computational grids [2, 6]. For this we apply the NC paradigm to a number of applications with which we have significant experience.

In the 1970s, the scientific computing community established the concept of a software library and introduced procedures for testing and disseminating such artifacts, which have evolved into international standards. Current information technologies (IT) allow the easy development and integration of GUI interfaces, domain specific textual and visual languages, visualization libraries, portable computational libraries, knowledge bases and other related technologies. These technologies already allow the user to exploit the power of the hardware resources while reducing the overhead of specifying and visualizing the results of a simulation. These developments have led to the concept of a Problem Solving Environment (PSE) that promises to provide industrial scientists and engineers with environments and seamless integration mechanisms allowing them to spend more time doing science and engineering rather than "computing". Now, the NC paradigm promises to put the PSE technology at the fingertips of any scientist and engineer anytime and anywhere. The first NC paradigm that we study is the so-called client/server computing. In this scenario, software usage is not viewed as a commodity but as service. The computational service provider will offer users all the resources needed to solve the problem in some "natural" form. In this context, we study the concept of client/server computing for PSEs and scientific libraries. This concept is demonstrated utilizing elements of the PELLPACK library and a "thin" [12] client-server WebPDELab.

The process of prototyping is part of every scientific inquiry, product design and learning activity. The new economic realities require the rapid prototyping of manufactured artifacts and rapid solutions to problems with numerous interrelated elements. This, in turn, requires the fast, accurate simulation of physical processes and design optimization using knowledge and computational models from multiple disciplines 
(multi-physics and multi-scale models) in science and engineering. The realization of rapid multidisciplinary prototyping is the new grand challenge. In these applications the software is often distributed geographically and the various groups involved have limited knowledge of all software components used to simulate the atomic elements involved in the prototyping of a composite artifact. In this application scenario, the natural computational resource is a "computational grid" or "The Net" that connects the needed distributed hardware and software resources used to simulate the elements of the artifact. We present the computational skeleton of a multi-physics application associated with the simulation of gas turbine engines and its implementation on a computational grid of heterogeneous computational resources, utilizing an agent based system known as Grasshopper [9] and Java based commodity software. Moreover, we discuss how these two implementations handle issues like heterogeneity, scalability, adaptability, and fault tolerance in the context of the targeted application scenarios.

The paper is organized as follows. Section 2 presents a detailed description of the thin client server WebPDeLab based on PELLPACK PSE and its libraries. Section 3 presents two implementations of a multi-physics application related to gas turbine engine simulation utilizing middleware technologies, the Grasshopper agent platform and a Java RMI middleware. In Section 4 we demonstrate two approaches for managing the software and computational resources based on locations of data, application code, and hardware. In these approaches, the code of interest is accessed as a service provided by a computational server. The user's data is sent to the server, the requested operations are performed, and the result is sent back to the initiator of the computation. We use the Java RMI middleware to implement remote computing with the ITPACK library and expand the PELLPACK PSE utilizing the NetSolve [4] middleware and its servers. Finally, Section 5 summarizes our observations.

\section{WEBPDELAB: A WEB-BASED THIN CLIENT-SERVER FOR THE PELLPACK PSE}

WebPDELab is a World Wide Web server that allows users to define, solve and analyze partial differential equation (PDE) problems using a comprehensive graphical user interface from any Java-enabled browser. The WebPDELab server is currently supported by a 32 CPU Intel cluster which allows users to solve PDE problems sequentially or in parallel. WebPDELab is the PELLPACK $[8,16]$ problem-solving environment 
implemented as an Internet-based client-server application. It provides access to a library of PDE solvers and an interactive graphical interface that support the pre-processing and post-processing phases of PDE computing. The PELLPACK software is implemented as a system of $\mathrm{X}$ windows programs and libraries, compiled on an i86pc SunOS 5.6 machine. WebPDELab displays the interface of the PELLPACK software within a Java-capable browser using the Virtual Network Computing [13] remote display system. The URL for the server is http://www.webpdelab.org. In this section, we give a brief overview of PELLPACK system and its implementation as a server utilizing the thin client/server technology.

\subsection{THE PELLPACK PROBLEM SOLVING ENVIRONMENT}

PELLPACK is a system that allows users to specify and solve PDE problems on a target computational platform and to visualize the solution. PELLPACK provides a graphical user interface for defining the PDE model and selecting solution methods (see Figure 3 ), and is supported by the Maxima symbolic system and well-known numerical libraries. The graphical interface is implemented on top of a very high level PDE language. Users can specify their PDE problem and its solution visually using the graphical interface or textually using "natural" language. PELLPACK has incorporated over 100 solvers of various types that cover most of the common PDE applications in 2 and 3 dimensions. In the PELLPACK system, a problem is represented by the PDE objects involved; PDE models or equations, domain, conditions on the domain boundary, solution methods, and output requirements. The PELLPACK interface consists of many graphical tools and supporting software to assist users in building a problem definition. A textual specification of these objects comprises PELLPACK's natural PDE language, and the language representation of each object is generated by the object editors/tools. The language definition of a user's problem (the .e file) is automatically passed to PELLPACK's language processor, which translates the problem into a Fortran driver program, and then compiles and links it with numerical libraries containing the userspecified solver methods. Sequential or parallel program execution is a one-step process; the program is executed on one or more machines in the supporting i86pc host cluster. Problem solutions are passed to the PELLPACK visualization system for solution display and analysis. 


\subsection{THE WEBPDELAB SERVER}

In response to the rising cost of managing distributed desktop based software, many organizations are revisiting a more centralized and managed computing strategy using thin client software and computing. Although this computing paradigm resembles a return to the days of mainframe computing, the new challenge is to handle the communication requirements for application software using sophisticated graphical user interfaces. Delivering raw screen pixels, however, requires bandwidth that most of the today's network environments cannot afford. Thinclient systems [12] use a remote display protocol to relay the display information from the server to the client efficiently. In [2] there is an evaluation of a variety of thin clients under various network conditions. We have selected the Unix-based AT\&T VNC [13] thin client-server system to implement PELLPACK PSE as the WebPDELAb web server because it is open-source and free unlike the other available platforms.

Users can connect to the WebPDELab site using any Java-enabled browser for information, demonstrations, cases studies and PDE problem solving service. A new PELLPACK session is initiated for each user that connects to the WebPDELab server, and a unique identification and private file space for the session are created. The file space is available until the user disconnects from the service, at which time the session is terminated and the user's files are deleted. Users may download files generated by PELLPACK to their own machines before terminating the session, and they may upload files to WebPDELab at the start of subsequent server sessions. When the server invokes the PELLPACK system software, the entire PDE problem-solving environment described in [5] is presented to the user. This environment was described briefly in Section 2.1 A detailed description of the functionality and operation of the PELLPACK software can be found in its User Guide that can be downloaded from the web site.

\subsection{THE WEBPDELAB INTERFACE}

The WebPDELab server is accessed from http://www.webpdelab.org. This web site is an instructional source for anyone interested in solving PDE applications. A collection of fully documented case studies is available at the site presenting step-by-step solutions of common PDE applications (flow, heat transfer, electomagnetism, conduction), with every user action and PELLPACK result described with images and detailed text. Users who request the PDELab problem solving service must first register with WebPDELab. After the user registration information is validated and the server connects to a host machine, WebPDELab presents 


\section{UPLOAD SERVER DOWNLOAD LOGOUT!}

Server accessed Thu Tob 17 13:46:19 EST 2000

Server brincusil.cs.purdue.edu: 1 is busy...

Server brincusil.cs.purdue.edu: 2 is available ...

Pacty to bunch Thu Fob 17 13:46:27 IST 2000. Yor UserD is ul34019

Click on the items above to control the PDELab Web Server

- UPLOAD upload PDELab fles from your machine. Upload fles before clicking on Server

- SERVER startup the PDELab System (password required)

- DOWNLOAD download PDELab files to your machine from your user directory

- LOGOUT! shutdown the PDELab Server

Figure 1 The WebPDELAB server with control panel in the top frame and instructions and connection information in the bottom frame

a framed HTML page, with a control panel in the top frame (see Figure 1) consisting of four options: Upload, Download, Server and Logout. The bottom frame contains the user identification number, host connection information, and instructions for using the options of the control panel in the top frame. At this point, the WebPDELab server has already created the user's directory space, so users can upload files to their directory using Upload. Generally, users upload PELLPACK problem definition files from previous WebPDELab sessions, such as .e files, mesh files and solution files. Users can upload up to 20 files to their assigned directory space, and files may no longer be uploaded once a user clicks on Server.

Download returns a listing of the user's directory contents. Files in this directory can be viewed or downloaded from the listing, but since users' directories are password protected, no other directories can be viewed or entered. Download is available throughout the user's session. Users should look here frequently during the session to check on PELLPACK generated problem, solution and trace files. The Server option invokes the password protected PELLPACK software. After the password is entered and verified, the top level window of the PELLPACK system appears in the bottom frame of the browser window as shown in Figure 2. A collection of sample problems has been placed in the user's directory, so users can load an example into the PELLACK session or begin their own problem definition. The session in Figure 3 is in the bottom frame of the WebPDELab server. The options of the control panel are still available in the top frame, but only the Download and Logout options 


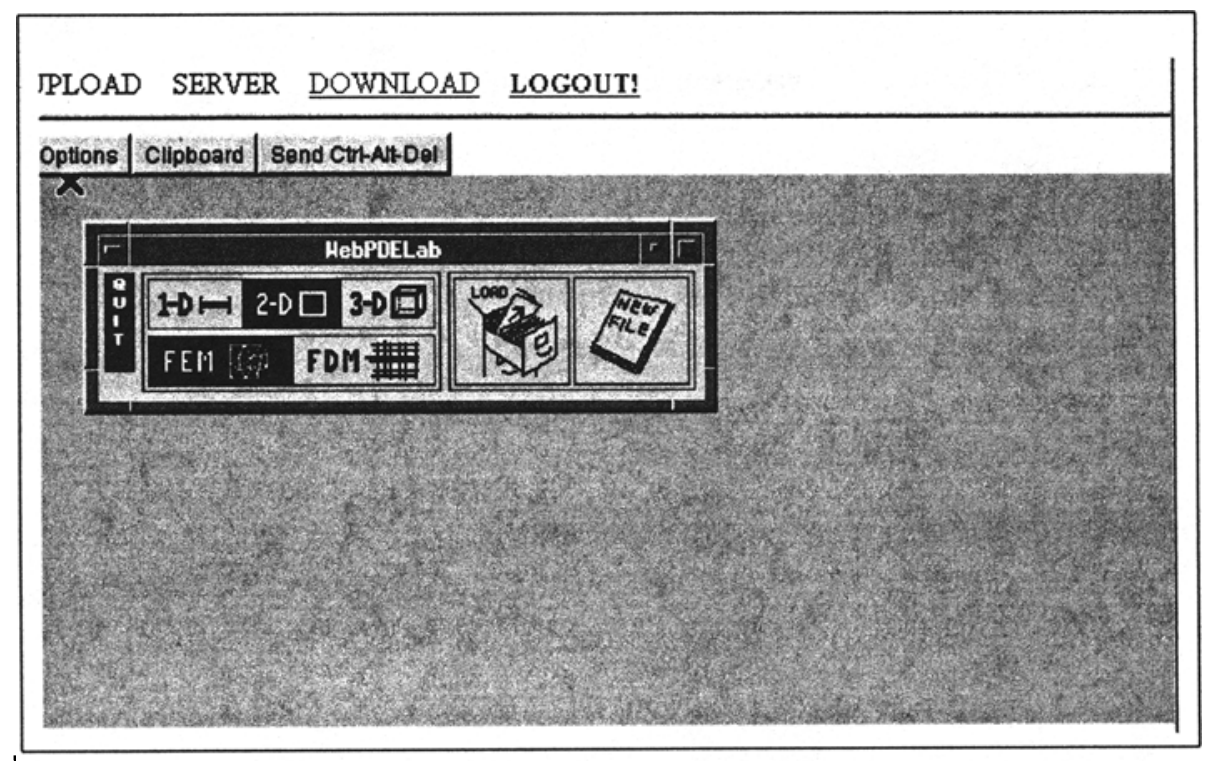

Figure 2 The PELLPACK top level window appears in the bottom frame of the WebPDELab browser window. It is ready for user interaction.

are enabled. The Upload and Server options remain disabled while the PELLPACK software is running in the bottom frame.

During the PELLPACK session, WebPDELab is passing the display of the remotely executing PELLPACK environment to the users browser window. The graphical interface displayed on the user's screen belongs to PELLPACK and is not described in this paper. When users click on Logout, the PELLPACK session is terminated and the user's directory is removed. WebPDELab traces all user activities from the start of the server session until its termination. Users files are secure from other users, but WebPDELab 'looks at' the contents of every file uploaded to WebPDELab or created by the user from within the PELLPACK system. Security mechanisms for the WebPDELab server and host cluster are discussed in Section 2.5.

\subsection{WEBPDELAB IMPLEMENTATION}

VNC is a remote display system that allows users to view a computing "desktop" environment from anywhere on the Internet using a wide variety of machine architectures. VNC consists of a server that runs the applications and generates the display, a viewer that draws the display on the client screen, and a TCP/IP connection between them. The server is started on the machine where the desktop resides, after which 


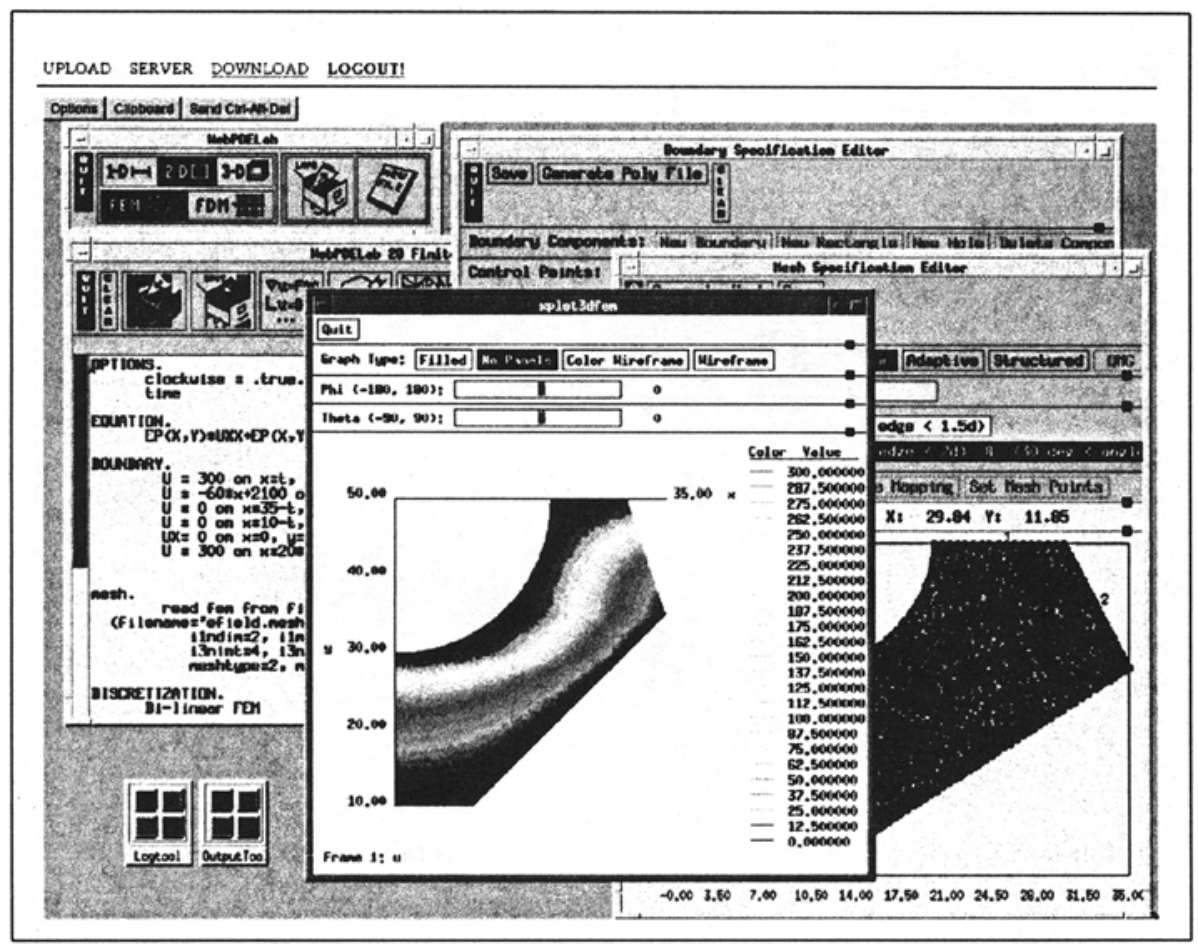

Figure 3 A PELLPACK session running inside the WebPDELab browser window.

any number of viewers can then be started and connected to the server. This allows the client user to access the applications, data, and entire desktop environment provided by the server. The viewer is a small, sharable, platform-independent, and stateless system that runs on the client machine. In the WebPDELab implementation, a new VNC UNIX server is started for each user who accesses the WebPELab web server from a Java-enabled browser (see Figure 4). The VNC Java viewer is started from the user's browser, allowing the user to display and interact with the PELLPACK environment, which consists of $\mathrm{X}$ windows programs and libraries compiled and running on the i86pc SunOS 5.6 host machines. Within this framework, any user worldwide who is connected to the Internet and has access to a Java-capable browser can run WebPDELab.

The WebPDELab manager is the collection of CGI scripts (Common Gateway Interface protocol for browser to server communication) that controls all user activity once the PDELab Server at the WebPDELab web site is selected. When a user accesses the server, the manager collects information on all currently running VNC servers from the host 
machines. The manager then asks the potential user to enter registration information, including a valid e-mail address. After the e-mail address is validated, a unique user id is generated for the new user, and a $\log$ file is set up to track registration information, user access/exit times, and user activities while running the PELLPACK software. The host machine with the lightest traffic is selected by the manager to run the VNC server, and subsequently the PELLPACK software. A protective client server application is used to launch the VNC server, so that users are never logged in to any machine in the host cluster. The VNC server startup invokes the PELLPACK system, and the manager creates the user directory, sends the control panel to the user, and monitors the user's interaction with the control panel options (Upload, Download, Server and Logout.)

Upload is implemented using copyrighted public domain code at http://stein.cshl.org/WWW/software/CGI (Lincoln Stein, 1998). The code has been modified to operate with the WebPDELab/VNC user directory privacy restrictions. Download is implemented as a standard link to the user's file space, but additional password security protects a user's assigned directory from all other users on the Internet. Server connects the VNC client user to the VNC server that has been instantiated for the caller on the selected host for a that server.

After control has passed to the VNC client, the manager waits for a VNC disconnect or a click on Logout. When signaled to start exit processing, the manager saves the trace of user activities to the log database, kills the VNC server, and removes the user's directory. The manager also checks all executing VNC servers periodically for sessions running longer than 24 hours, and these sessions are terminated. When the manager has finished exit processing, control is returned to the WebPDELab home page.

\subsection{WEBPDELAB SECURITY ISSUES}

When a user logs into the WebPDELab server, a CGI script is executed which generates the unique user id and requests one of the cluster host machines to invoke a VNC X-server. The WebPDELab CGI scripts reside on an isolated machine dedicated to serving CGI requests. This machine has no NFS-mounted disks, therefore an attacker attempting to take advantage of vulnerable CGI scripts is locked into the cgi-bin directory and cannot gain access to any other machines or disks. All parameters passed to WebPDELab CGI scripts are scanned to ensure they contain precisely the expected values (argument number, length and contents), else the request is terminated. The cluster machines lis- 


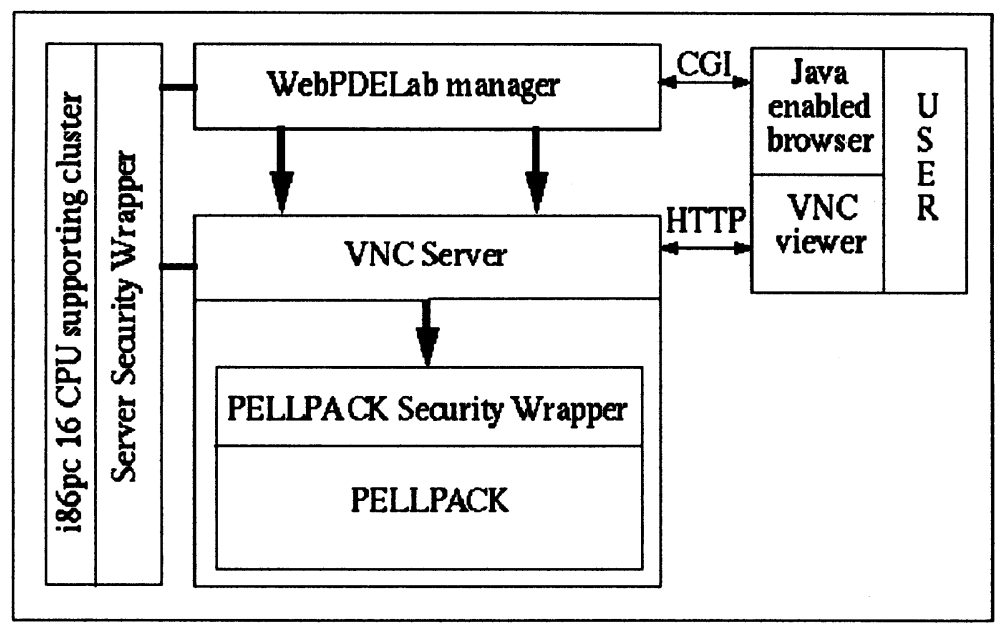

Figure 4 Implementation of the WebPDELab server.

ten on a fixed port for startup requests from the CGI machine. If an attempt is made to connect to this port that does not originate from the CGI host, the connection is immediately terminated. All cluster machines run a daemon that listens for socket connections on a specified port and spawns a child process to serve the request, while the parent continues to listen for other connections, so that requests can be served simultaneously. A client program is invoked by the CGI script to contact the cluster machine, and requests that a new VNC X-server be launched. The client may only specify the VNC X-server startup parameters, since the launching of the VNC X-server binary is hard-coded in the configuration file of the daemon serving requests originating from the CGI host. The VNC server itself is protected by a challenge-response password scheme.

The cluster machines run the VNC X-server, as owned by a dedicated account whose root directory is the account's home directory (using the Unix maintenance chroot command). All the required binaries are located in the chroot directory. If a user discovers vulnerabilities in one of the cluster machines, the user is locked into the home directory of the account, and is unable to cause harm to other accounts or disks. In order to protect the machine from unauthorized Fortran code inserted by a user into the PELLPACK .e file, specialized filters have been built into the original PELLPACK system. The PELLPACK language processor initially restricts the location of Fortran code to specialized segments within the PELLPACK problem definition file; these segments are now 
re-parsed by filters that check inserted Fortran statements for unauthorized code.

Every user is provided with a unique directory for uploading and downloading files, thus facilitating the option of saving and retrieving material. The CGI script creates this directory after the registration information is entered and validated. User's directories are password protected, securing each user from all other users. Every user file, however, is opened and checked by WebPDELab for legal content as it is uploaded or saved by the user from inside PELLPACK.

\subsection{WEBPDELAB FEATURES AND ISSUES}

The significant benefits that can be obtained from the implementation of the WebPDELab server are:

- Generality. Any machine connected to the Internet can use the PELLPACK environment without concerns about language or machine compatibility.

- Interaction. Users can specify the PDE with normal interaction speeds for the client machine, since data entry is done locally. The amount of code exported to support the user interface is substantial (several megabytes), but it is only a small fraction of the PELLPACK system. If the user has no graphics capability, then the text based interface tools must be used; these are less convenient but still practical to use.

As the PDE problem is being specified, information is sent to the server. The server might request additional information but once the problem is completely specified, it is solved on the server's host machines. After the PDE is solved, the user can either view output generated by the server or request that the solution (normally a large data set) be returned for local use.

- Access to high performance computers. Any user can access machines with sufficient power to solve the PDE problem. Even if the solution is too large to be sent to the user (or if there are no local visualization tools), the solution can be explored over the Internet.

- No code portability problems. Users do not need to have the code in the local machine language, since the software infrastructure operates only on the server's host machines.

There are several concerns and technical issues involved in the service provided by WebPDELab which we now discuss: 
- Performance of the user interface. There is a clear trade off in user interface performance between exporting code to the user's machine and executing code on the server. Our initial prototype showed that communicating each mouse click back to the server for processing provides unsatisfactory interactive performance due to network delays. Our analysis indicates that almost all of the interaction can be run locally by exporting a moderate amount of code. The user interface does use tools that are both time consuming to execute and which are too large to export. Examples are Maxima (used to transform mathematical equations) and domain processors (used to create meshes or grids in geometric domains). These tools usually require pauses in response even without a network and the added delay due to networks is unlikely to be significant.

- Security for the server. While we control the material received from a user, the server is clearly subject to attack. We place the server on a separate subnet and access licensed software through a gateway. Since we know exactly what is to be sent via an RPC, it is possible to protect this licensed software. Even if a user succeeds in becoming "root", access to other machines is not possible. Of course, network file systems and similar tools are not used. Our process of "registering" users when we give them accounts provides us with a chance to screen users before providing them access to WebPDELab.

- Security for the user. This requires each user to be completely isolated from all others. Each user on the server runs in a virtual file system using a login with no access privileges. Thus, each user appears to have the entire machine, and the protection mechanisms between machines, protects users from one another. This approach provides security at the cost of using much more memory than normally necessary.

- Software ownership and fair use. We prevent the copying of software by placing, if necessary, source code on another machine or another network and using secure RPC.

- Payment for computing services. The WebPDELab server is provided free to users as well as time on associated servers used for security purposes. We do not foresee a need to charge users for time on these machines. If large numbers of users contend for service then they will be queued and the cost of the servers is clearly limited. 


\section{HETEROGENEOUS DISTRIBUTED COMPUTING VIA LAN/WAN}

High-performance networks have already become an integral part of today's computing environments. This new capability allows applications to evolve from static entities located and running on specific hosts to applications that are spread across Internet or Intranet based networks. In a networked-based computing environment or computational grids the fundamental research problem could be viewed as the development of an infrastructure that supports the construction of applications by composing dynamically application-specific components, mostly "legacy", that are identified and linked together at run-time in a secure and robust way. In this paper we utilize and evaluate various software technologies and architectures to solve the above problem in the context of a multi-physics application [11]. We expect the experience gained by this study to allow other application researchers to realize the capabilities and limitations of existing technologies in their attempt to take advantage of a networked-based environment in their application domain. In [2] several back-end systems are reviewed to implement the networked-based scenario for different type of applications. In this study we experiment with the agent based Grasshopper system and with the Java RMI middleware. In this section, we give a short overview of Grasshopper and describe the design of the gas turbine engine model PSE (GasTurbnLab) and its two implementations.

\subsection{GRASSHOPPER AGENT SYSTEM}

Grasshopper [9] is an agent development platform that supports distributed agent-based applications. The platform provides a base for communications services, mobile computing power and dynamic information retrieval. It is essentially a mobile agent platform that is built on top of a distributed processing environment, integrating the traditional client-server paradigm and mobile agent technology. The primary feature of the Grasshopper platform is its location independent computing, driven by the ability to move agents between different systems. It is powerful tool that facilitates the creation of agents, transparently locating them and controlling their execution. These agent-based applications are interoperable with other agent systems that are MASIF (Mobile Agent System Interoperability Facilities) compliant. The Grasshopper Distributed Agent Environment is composed of regions, agencies and agents. At the top of the hierarchy is the region that manages the distributed components in the Grasshopper environment. Agencies and agents are associated with a particular region. Each region has a registry 
that maintains information about all components associated with it. An agency is the runtime environment for mobile and stationary agents, providing the functionality to support agent execution. The agency is responsible for a number of services including: (1) communication services for all remote interactions that take place between Grasshopper components, their movements and transport. Interactions can be performed by CORBA, IOP, RMI or plain socket, (2) registration service to track all currently hosted agents, (3) management services that monitor external control of agents, (4) transport services for migration of agents from one agency to another, (5) security services for protecting remote interactions and agency resources, and (6) persistence services for enabling the storage of agents for possible recovery. Agents are computer programs characterized by a set of attributes. They can be mobile or stationary. Mobile agents move from one location to another within a region to take advantage of local interactions, and thus are capable of reducing network loads by migrating. Stationary agents are associated with a particular location only and are incapable of migration.

\subsection{THE GAS TURBINE ENGINE MULTI-PHYSICS MODEL}

The gas turbine engine is an engineering triumph. It has more than 1,300 parts with rotational speeds to $16,000 \mathrm{rpm}$ for axial and $50,000 \mathrm{rpm}$ for radial flow components. For aircraft applications, it operates with maneuver loads of up to $10 \mathrm{~g}$, with flow path pressures and temperatures to 40 atmospheres and $1400 \mathrm{~F}$. The important physical phenomena take place on scales from 10-1000 microns to meters. A complete and accurate simulation of an entire engine is enormously demanding; it is unlikely that the required computing power, simulation technology or software systems will be available in the next decade. The primary goal of our research in this area is to advance the state-of-the-art in very complex scientific simulations and to validate the simulation results. Specifically, we consider simulating the compressor-combustor coupling in a gas turbine engine. We view the application as a set of collaborating physical models. The hardware infrastructure assumed for these simulations consists of a computational grid involving a SP-2, 128 PC cluster running Solaris, and SGI Origin 2000 with 32 CPUs. Next, we consider the utilization of the Grasshopper agent system to implement GasTurbnLab. 


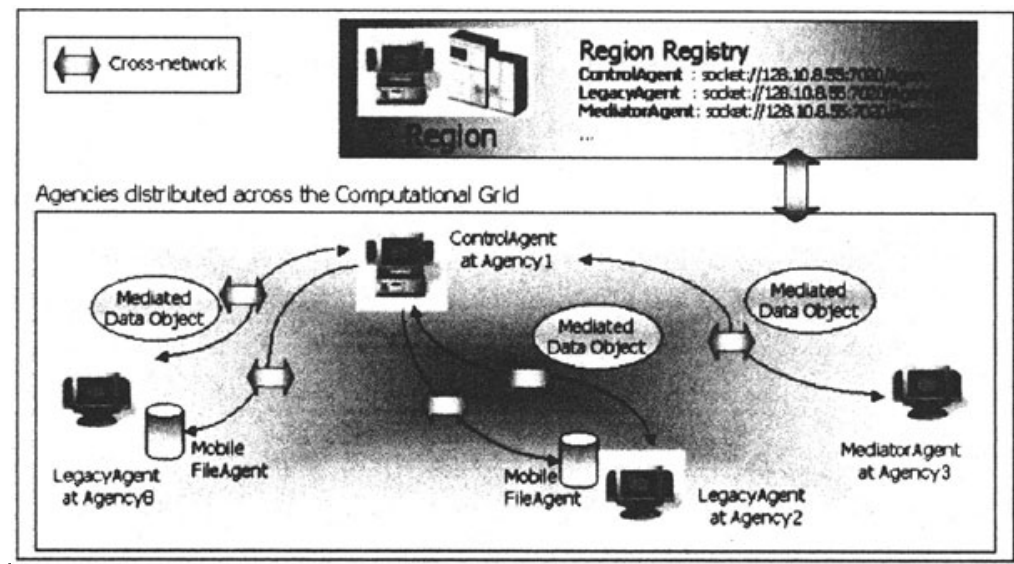

Figure 5 The grid computing scenario for the Grasshopper implementation of the GasTurbnLab simulation.

\subsection{THE GRASSHOPPER IMPLEMENTATION OF THE GAS TURBINE ENGINE MODEL}

The Grasshopper environment supports the traditional client-server structure. GasTurbnLab servers are Grasshopper agents which provide computational service and data visualization. The GasTurbnLab client agent controls the entire simulation process by launching/terminating the server agents, managing their interactions and facilitating the crossnetwork asynchronous communication between computational and control data. GasTurbnLab is implemented using four classes of agents: the simulation control agent (SCA), the visualizer agent (VA), the legacy code agents (LCA) and the mediator agents (MA). The SCA is the client which requests and manages the services of the remaining classes of agents. The VA is a server which receives solution data (velocity, speed, density, energy, pressure) for one or more engine parts, and renders it graphically using the Iris Explorer data visualization system [10]. The LCAs are the computational agents which simulate the engine; each agent encapsulates an established legacy code targeted for a specific engine part. The GasTurbnLab model requires the LCAs to communicate their boundary data (via the SCA) to one or more MAs. The MAs encapsulate the mediation codes which are responsible for adjusting and resolving interfaces (represented by the boundary data) between neighboring engine parts, each of which is simulated by one LCA. The GasTurbnLab SCA can handle any number of engine parts, that is, any number and type of communicating legacy code and mediator agents. 
The initial challenge was to create a template procedure for embedding legacy Fortran codes into the server agent structure. The resulting LCAs could then exercise control over the Fortran code and enable data flow by starting up the legacy code, pausing after each iteration to communicate the required boundary data to the SCA, receiving the mediated boundary data in return and continuing with the next iteration. Two legacy codes have been encapsulated as LCA agents: Ale3D, an advanced CFD code for simulating turbines [7] and Kiva, an advanced combustion simulation code [1]. The template procedure requires the legacy code, which generally starts out as a Fortran executable, to be transformed into a C-wrapped legacy code library as follows: (1) change the Fortran main to a subroutine, with command line arguments passed as parameters, (2) start the Fortran subroutine as a thread from the $\mathrm{C}$ wrapper routine, (3) define $\mathrm{C}$ structures to hold the data representing boundary information, (4) write a $\mathrm{C}$ data transfer routine to copy boundary data back and forth between the $\mathrm{C}$ and Fortran data structures, (5) insert a call to the $\mathrm{C}$ data transfer routine in the Fortran iteration loop, and (6) insert control code in the $\mathrm{C}$ wrapper to sleep/wake the $\mathrm{C}$ data transfer routine, thus effectively controlling the pause and restart of the Fortran iterations. The changes to the Fortran code are minimal. The $\mathrm{C}$ wrapper is defined with a JNI [14] interface to the Java agent code, and the C boundary data is passed up through the JNI interface parameters to the Java agent. The Fortran code and two $\mathrm{C}$ routines are compiled together as a legacy library which is loaded into the Java LCA server when it is instantiated. The Java legacy agent starts the $\mathrm{C}$ wrapper and waits for the JNI object containing the boundary data. When the object is received, the agent serializes it and communicates it to the SCA. The SCA returns a mediated data object to the agent, which copies it into a JNI object and passes it to the C wrapper. The SCA is also responsible for passing the legacy agent a termination signal.

Mediator agents are customized Java programs that are easily encapsulated as Java agents. The interface relaxation code is heavily dependent on the legacy codes and data types which are being mediated. The mediator code is wrapped by agent code which handles the boundary data object communication between the MA and the SCA.

The GasTurbnLab project has successfully implemented an AleAgent (LCA) for simulating both rotor and stator engine parts (an engine part is also called a domain), a KivaAgent (LCA) for simulating a combustor, and a MA to mediate boundary data. The MA supports mediation between two Ale3d domains (stator and rotor) and between Ale3d and Kiva domains (stator and combustor). The first prototype was a two domain Ale3d stator-rotor simulation. We assume a running Grasshop- 


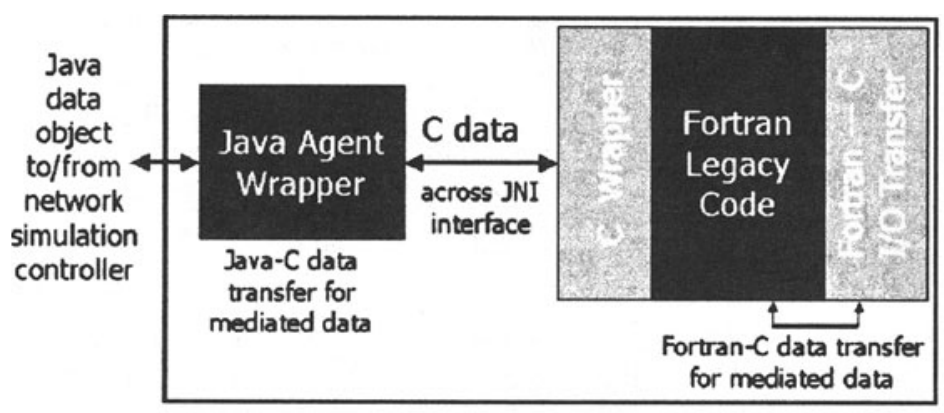

Figure 6 Implementation of the Legacy Code Agent.

per region with 3 or 4 registered agencies on machines belonging to the GasTubnLab computational grid (see Figure 5). Two AleAgents, an MA and a VA are loaded into (any combination of) the agencies and automatically registered with the region. The SCA is loaded into an agency and started as a stator-rotor controller. The SCA first looks up the registry to determine the availability of the necessary servers. It then supplies the two AleAgents with grid and parameter information, and the server agents commence execution. After the encapsulated legacy code finishes one cycle of computation, the boundary data is copied upward from the Fortran code to the C-wrapper to the JNI object to the Java agent. The SCA receives this data from the AleAgents, then filters and serializes the data for the MA. The MA is called with this data and, after running the interface computations, it returns the mediated data to the SCA. The SCA restarts the AleAgents with the mediated data. This process continues until the termination signal is given. The VA is launched with solution data from both domains, and users can interactively choose which domains and solution data to display. This prototype simulation has run thousands of iterations and the solution output data has been verified.

\subsection{THE JAVA RMI IMPLEMENTATION OF THE GAS TURBINE ENGINE MODEL}

The presence of the Grasshopper platform simplifies several aspects of the implementation, such as the asynchronous communication and the agent management. However, the current implementation of the gas turbine engine simulator does not use Grasshopper for agent migration or persistent storage. Thus, we have considered an implementation that uses only Java RMI. A direct Java implementation gives us better insight and control over our system, at the cost of programming ourselves part of 
the functionality provided by Grasshopper, mainly asynchronous remote method calls.

Our Java RMI implementation of the gas turbine model was based upon the Grasshopper design. Thus, the software interfaces of the model modules are either the same as those in the Grasshopper implementation, or (in the worst case) equivalent to them. The control flow is almost identical. For keeping track of the available agents we created a simple custom registry that itself uses the standard RMI registry provided by the Java SDK. In the two-domain problem described for the Grasshopper implementation, we first start our custom registry. All the agents (AleAgents, MAs, VA) are registered in the same custom registry. The SCA looks up the custom registry for available agents. If enough agents are available, the SCA supplies the AleAgents with parameters, and the first cycle of computation begins. Hereafter the execution and data copy chain between the various agents and wrappers continue in the way described in Section 3.3 until the computations are complete. This implementation has shown that pure Java technology can be easily utilized to implement distributed computations. In the near future we will compare the efficiency of the two implementations.

\section{DISTRIBUTED SCIENTIFIC LIBRARIES AND PSES}

In this section we demonstrate two middleware approaches to managing the software and computational resources with respect to data location, application code, and hardware. The approaches are based on the so called remote computing or traditional client-server approach, such as that provided by CORBA. The code of interest is accessed as a service provided by a computational server. The user's data is sent to the server, the requested operations are performed, and the result is sent back to the initiator of the computation. In this computational paradigm, there is generally a tight binding between the software and the computational resources on which the software runs. We use the Java RMI middleware to implement remote computing with the ITPACK library, and expand the PELLPACK PSE utilizing the NetSolve middleware and its servers.

\subsection{A WEB-BASED ITPACK LIBRARY SERVER}

In this section we describe the implementation of ITPACK library as a remote computational server utilizing the Java RMI technology. ITPACK library implements a number of iterative methods for solving finite element and difference equations resulting mainly from the 


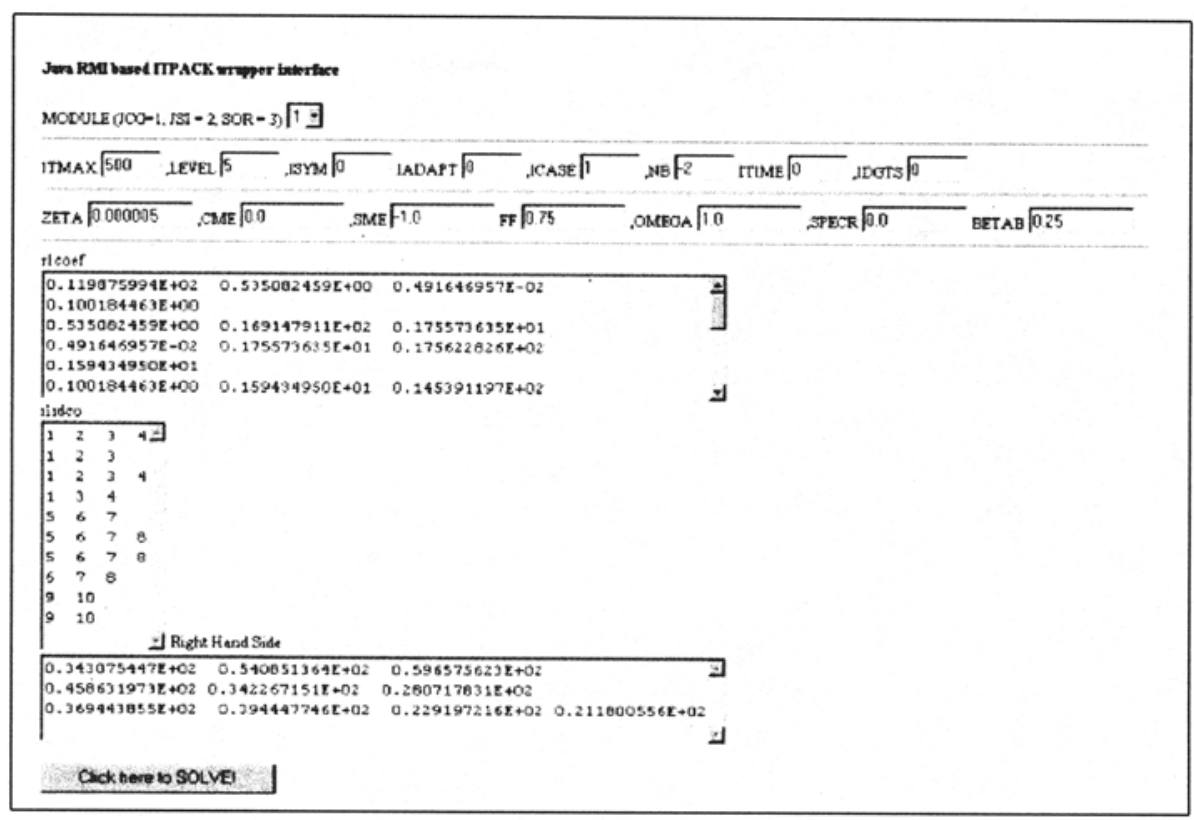

Figure 7 Java RMI based ITPACK wrapper interface. The user selects the module and its parameters, inputs the matrix in a sparse storage scheme and inputs the right-hand-side. The system returns the solution.

discretization of elliptic boundary value problems. Java's RMI capability allows programmers to invoke object methods residing on a remote machine. More importantly, it allows the serialization of objects, thus enabling the client and server to reference remote objects. The Fortran code of the ITPACK library routines were wrapped with a simple $\mathrm{C}$ function that accepted relevant parameters in order to invoke the appropriate ITPACK routine. This $\mathrm{C}$ wrapper was compiled into a library, which was loaded by the top-level Java RMI server method. The Java client and server programs shared common understanding of the object to be referenced. Such an object was initialized by the client program and then passed over to the remote RMI method, which then called the $\mathrm{C}$ ITPACK wrapper routine. After the execution of the RMI method, the variables of the client program object contain the computed solution. In addition, we implemented a web-based interface to the ITPACK server, shown in Figure 7. It consists of a simple HTML form that posts the values of the parameters entered by a user to the Java RMI client program, and outputs its result in the web browser. 


\subsection{A NETWORK-BASED PELLPACK PSE}

In this section we consider a scenario for PELLPACK where either code is shipped to the requesting location (executing in the requester's environment using "local" data) or the code of interest is accessed as a service provided by a computational server. In the second paradigm, the intermediate PELLPACK data is sent to the server, the requested operations are performed, and the result is sent back to PELLPACK.

The first computational paradigm could be implemented by a network file system to support the sharing of well-defined software libraries with PELLPACK across LAN and WAN computational environments. Many different versions of network file systems exist, e.g. Sun's Network File System (Sun NFS), the Andrew File System (AFS) [15] and the Coda file system [3]. The Sun NFS is primarily designed for local area networks while AFS and Coda were developed with a wide area network application in mind. As a consequence, different approaches are employed for caching, replication, and recovery, which affect performance (especially over slow links) well as data integrity. The most crucial difference, however, lies in the level of security offered. Specifically, AFS and Coda employ rigorous authentication protocols, thereby ensuring that the server will reject "unauthorized" requests. On the other hand, in the off-the-shelf Sun NFS, the client authenticates the user while the server accepts and executes incoming client requests. It is thus (theoretically) possible to construct "man in the middle" attacks, circumventing the proper client authentication procedure.

When NFS or AFS exported shares are mounted locally, the end-user can deal with them as if they are contained in another directory present on their local disk. Since PELLPACK makes use of various libraries to perform its computations, these libraries can be stored at various machines on a WAN or LAN and exported for use by local machines. The main advantage of such an approach is that the PELLPACK program does not recognize the remote existence of these libraries, and makes use of them as if they were available locally.

The second computational paradigm is currently implemented by utilizing the NetSolve Project. NetSolve is a RPC based client/agent/server system that supports remote access to both hardware and software computational resources distributed across a network. NetSolve responds to a user's request for service and solves the problem and returns the solution to the user. NetSolve provides secure yet flexible mechanisms for cooperation and control between resources, processes, data and users. NetSolve has implemented interfaces in Fortran, $\mathrm{C}$ and Matlab for a large number of computational software components. 


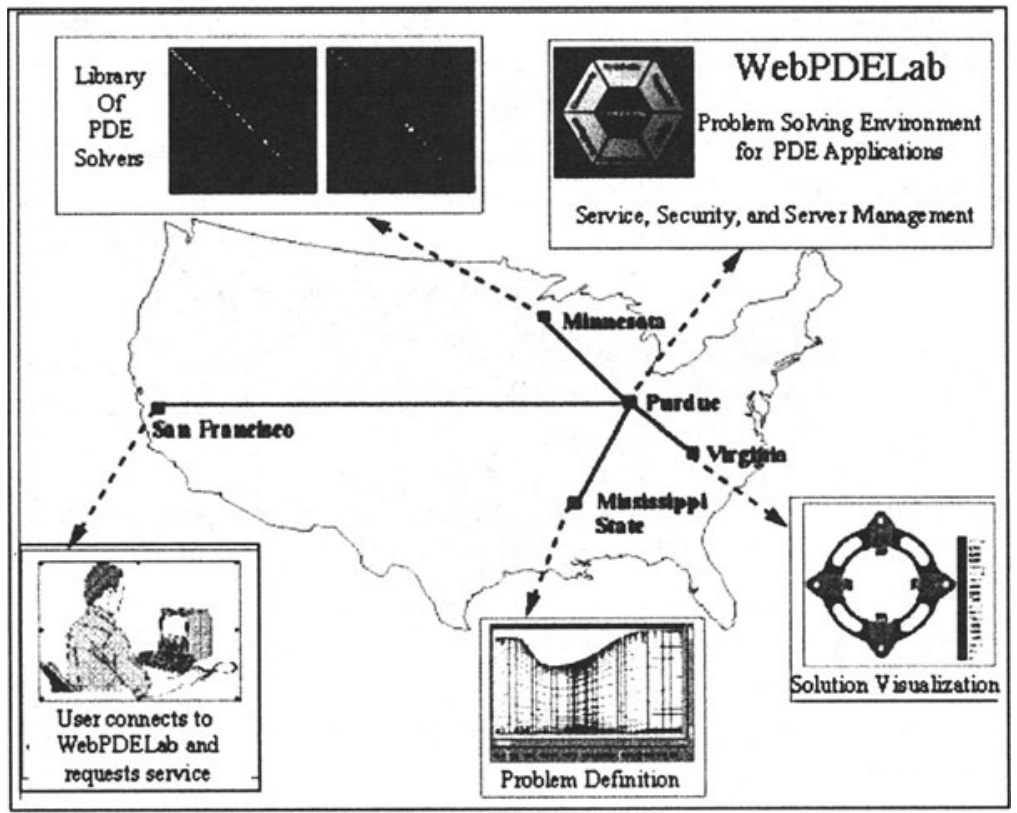

Figure 8 A networked computing view of the PELLPACK PSE supported by a distributed file system and/or NetSolve

The open architecture of the WebPDELab PSE supports the easy integration of software components used in the PDE problem solving process. The WebPDELab framework that has been implemented for the NetSolve interface allows WebPDELab users to specify any of the computational components available through NetSolve (i.e., components which are related to the PDE solution process) from within WebPDELab's graphical environment. This framework opens up the entire NetSolve environment for use by the WebPDELab PSE. It provides:

1 The running server and agent processes required by the NetSolve server. NetSolve requires users to download, compile, link and configure the system that comprises NetSolve's agent/server control, communication and hardware/software interfaces. This software is installed and running on WebPDELab's supporting i86pc machine cluster, and allows WebPDELab users to access the NetSolve system without downloading and installing the NetSolve system on their own machines.

2 A library of interface routines handling the conversion of WebPDELab data structures (matrices, parameters, etc) to NetSolve problem definition data structures and back. The NetSolve system 
requires users to write their own Fortran, $\mathrm{C}$ or Matlab code to define the problem and set up the data structures in preparation for a call to the NetSolve software/hardware resources. This process has been automated in WebPDELab by allowing users to select algorithms (such as linear solvers) and their parameters graphically, and then providing data conversion and NetSolve communication services without any intervention by the user.

3 A call to NetSolve requesting service for the user-specified algorithm, with the call setup and problem definition parameters taken care of automatically by the WebPDELab server.

The WebPDELab-NetSolve integration framework supports the inclusion of new NetSolve algorithms as a simple two-step process which is handled by the WebPDELab support group. First, a simple conversion routine is written to transform WebPDELab data structures to NetSolve structures and added to the WebPDELab interface library. Second, the name of the new algorithm and a list of its parameters are inserted in WebPDELab's language processor and in the graphical algorithm specification module. Since only NetSolve software related to the PDE problem solving process makes sense in the WebPDELab environment, these are the NetSolve algorithms that have been integrated. The WebPDELab-NetSolve integration has been tested for a collection of sparse linear solvers from the numerical software resources provided by NetSolve.

\section{CONCLUSION}

"Many a mickle makes a muckle" observes a recent article in Economist magazine. It predicts that harnessing the spare computing capacity scattered around a zillion desktops through the Internet soon will be reality and worth real money. Already there are several commercial and research projects trying to exploit this "something-for-nothing" idea. Distributed computing over the Internet is known by many terms, such as network computing, meta-computing, seamless scalable computing, networked based computing, net-centric computing, grid computing and internet computing. The realization of network computing paradigm depends on several developments that include middleware for heterogeneous, fault tolerant and secure computing in a computational grid involving thousands of machines, partitioning and scheduling of large computational problems, and intelligent management of all these resources. In this paper, we argue that network computing is feasible with existing software and algorithmic technologies, even for computations based on "legacy" codes. We have shown that "legacy" scientific 
PSEs and libraries can be used in a network computing setting. We have experimented with partitioning, mapping, and execution of a large-scale multidisciplinary application simulated by "legacy" software across a network of resources utilizing an agent based middleware (Grasshopper) and pure Java technologies. This experience confirms the feasibility of the network computing paradigm and its potential.

\section{Acknowledgments}

This project was partially funded by the National Science Foundation (CCR9202536, ASC-9404859, CCR-8922537, CCR-9311486), the US Department of Energy ASCI program, contract LG-6982, Intel Corporation.

\section{References}

[1] A. A. Amsdem. KIVA-3V, A block-structured KIVA program for engines with vertical or canted valves. Technical Report LA-13313MS UC-1412, Los Alamos National Laboratory, 1997.

[2] M. Baker, R. Buyya, and D. Laforenza. The Grid: International efforts in global computing. Proceedings of International Conference on Advances in Infrastructure for Electronic Business, Science,and Education on the Internet (SSGRR'2000), to appear, July 31- August 6, 2000.

[3] P. Braam, M. Satyanarayanan, M. Ebling, J. Raiff, and J. Harkes. The coda distributed file system user and systems administration manual, May, 2000. Available via World Wide Web at http://www.coda.cs.cmu.edu/.

[4] H. Casanova and J. Dongarra. Applying netsolver's networkenabled server. IEEE Comp. Sci. \& Engr., 5, No. 3:57-67, 1998.

[5] A. C. Catlin, S. Weerawarana, E.N. Houstis, and M. Gaitatzes. The PELLPACK User Guide . Technical Report, Dept. of Computer Sciences, Purdue University, to appear, 2000.

[6] Ian Foster and Carl Kesselman. The grid: The blueprint for a new computing infrastructure. 1999.

[7] J. Hallquist. Theoretical manual for dyna3d. Technical Report UCID 19401, Lawrence Livermore Laboratories, 1983.

[8] E.N. Houstis, J.R. Rice, S. Weerawarana, A.C. Catlin, M. Gaitatzes, K. Wang, and P. Papachiou. PELLPACK: A Problem Solving Environment for PDE-based Applications on Multicomputer Platforms. ACM Trans. on Math. Soft., 24, No. 1:30-73, 1998.

[9] IKV++. The grasshopper agent platform, 2000. Available via World Wide Web at http://www.ikv.de. 
[10] IRIS Explorer Center. Iris explorer toolkit, 2000. Available via World Wide Web at http://www.nag.com/IEC.

[11] S. Markus, E. N. Houstis, A. C. Catlin, J. R. Rice, P. Tsompanopoulou, E. Vavalis, D. Gottfried, Ke Su, and G. Balakrishnan. An agent-based netcentric framework for multidisciplinary problem solving environments (mpse). Computational Engineering Journal, to appear., 2000.

[12] J. Neih and S. Yang. Thin is in, July, 2000. Available via World Wide Web at http://www.columbia.edu/ nieh/research/thin.

[13] T. Richardson, Q. Stafford-Fraser, K. R. Wood, and A. Hopper. Virtual Network Computing . IEEE Internet Computing, 2, No. 1:33-38, 1998.

[14] Sun Microsystems. The java native interface. Sun Microsystems, Inc. Java Software, 1999.

[15] Transarc Corp. The afs file system in distributed computing environments, 1996. Available via World Wide Web at http://www.transarc.com/Product/EFS/AFS/.

[16] S. Weerawarana, A. C. Catlin, S. Chui, C. Crabill, E.N. Houstis, S. Markus, and J.R. Rice. PDELab: An object-oriented framework for building problem solving environments for PDE based applications. pages 79-92. RogueWare Software, Corvallis, OR, 1994. 


\section{DISCUSSION}

\section{Speaker: Elias Houstis}

Vladimir Getov : The second project as presented in your talk makes extensive use of agents. Is there a specific reason for that?

Elias Houstis : The agent computational paradigm fits intellectually better the MPSE concept and the networked mathematical models for simulating multidisciplinary applications like the gas turbine engine. Moreover, it provides a higher level middleware for implementing heterogeneous distributed computations. We have a Java RMI implementation of the GasTurbnLab that works equally well for small number of components. It remains to be seen whether the two implementations will scale in efficient way.

Morven Gentleman : Can you provide an estimate of the integration effort that it took to make each of your three examples operational?

Elias Houstis : The WebPDELab is a one work-year effort. The GasTurbnLab is a five work-year effort. The distributed WebPDELab is less than a one-half work-year effort.

Margaret Wright : Could you comment on the issues arising from the user interface? Some users want to use an advanced graphical user interface, but there is also a need for file-based input (for data), and perhaps some specialized modeling languages. What approaches have you taken?

Elias Houstis : We have been experimenting with graphical, very highlevel domain-specific languages, and visual programming languages in the domain of PDEs. We found to be in general useful. In the case of GasTurbnLab, we have utilizing IRIS Explorer commercial product to implement its interface. It supports visual programming and graphics.

Ronald Boisvert : In GasTurbnLab you are gluing together many modeling components, each of which is very complex. As you scale up to a large number of these, how do you verify that you are getting the right answers to the problem?

Elias Houstis : We are using specialized software modules/agents, called mediators, among the various components that measure physical and mathematical indicators, implement engineering intuition, and make decisions about the analysis/design iterative process.

Ivor Phillips : Since you have opened your systems to the whole world there is a concern that someone may submit a problem so large that it will consume all of your computing resources for weeks (or more). Do you have facilities for checking submitted problems for such issues and rejecting them? 
Elias Houstis : We monitor WebPDELab user activities and we could take appropriate action at any time. We have not faced this problem so far. Thus, we have not instituted policies and/or facilities to avoid or discourage such situations.

Richard Fateman : Do you check for plausibility of input at the interfaces between the numerous distributed modules in your applications? Elias Houstis : We filter the input only for unwanted code. Usually, users consult us when they have problems. 